\title{
Radial versus Combined Shockwave Therapy in the Management of Proximal Hamstring Tendinopathy: Similar Functional Outcomes in Running Cohort
}

\author{
P. H. Yun ${ }^{1}$, S. DeLuca ${ }^{2}$, D. Robinson², A. Park ${ }^{2}$, C. Rosenberg ${ }^{3}$, M. J. Kohler ${ }^{4}$, \\ A. S. Tenforde ${ }^{2}$ \\ 1 Department of Medicine, Massachusetts General Hospital/Harvard Medical School, Boston (MA), U.S.A. \\ 2 Department of Physical Medicine and Rehabilitation, Spaulding Rehabilitation Hospital/Harvard Medical \\ School, Charlestown (MA) U.S.A. \\ 3 Boston University, Boston (MA), U.S.A. \\ ${ }^{4}$ Division of Rheumatology, Allergy, and Immunology, Massachusetts General Hospital/Harvard Medical School, \\ Boston (MA), U.S.A.
}

\author{
CORRESPONDING AUTHOR: \\ Adam S. Tenforde \\ Department of Physical Medicine \\ and Rehabilitation \\ Spaulding Rehabilitation Hospital/Harvard \\ Medical School \\ $3001^{\text {st }}$ Avenue \\ Charlestown (MA) 02129, U.S.A. \\ E-mail: atenforde@mgh.harvard.edu
}

DOI:

10.32098/mltj.04.2021.18

LEVEL OF EVIDENCE: 4

\section{SUMMARY}

Background. Proximal hamstring tendinopathy is a common cause of gluteal pain. Extracorporeal shockwave therapy may be an effective treatment in proximal hamstring tendinopathy. However, published outcomes are primarily limited to evaluating radial shockwave, and the use of combined treatment (focus and radial treatment) and outcomes for management in runners are not well described. The purpose of this report was to characterize functional outcomes using radial and combined shockwave in the management of proximal hamstring tendinopathy in runners. We hypothesized that runners who received R-SWT or C-SWT would experience improvement in functional outcomes using the VISA-H.

Methods. This study is a quality improvement initiative evaluating clinical outcomes in a single outpatient clinic. Sixty-three runners (mean and standard deviation for age and duration of symptoms $42.8 \pm 14.7$ years and $16.9 \pm 23.8$ months, respectively), were identified as receiving treatment for management of unilateral or bilateral proximal hamstring tendinopathy. Patients were treated with either radial $(n=40)$ or combined shockwave therapy $(n=$ 23) using similar post-procedure protocols, including recommendations to complete physical therapy exercises of core and lumbopelvic stabilization with gradual progression to eccentric strengthening of the hamstring complex. Victorian Institute of Sport Assessment - Proximal Hamstring Tendons (VISA-H) was used to assess treatment outcomes, evaluated as differences between treatment cohorts by mean values from baseline to follow-up after shockwave treatment. The number in both treatment groups who met minimal clinical important difference (MCID) was defined as a gain of 22 points or more on VISA-H.

Results. Patients in both radial and combined shockwave groups received a similar average number of treatments $(5.0 \pm 2.2$ vs $5.2 \pm 1.9 ; \mathrm{p}=0.740)$. The radial and combined shockwave groups' mean VISA-H scores were similar at baseline $(39.4 \pm 17.4$ vs $40.7 \pm 17.0)$ and achieved similar final scores $(62.6 \pm 19.7$ vs $63.4 \pm 21.3 ; \mathrm{p}=0.812)$, and nearly all had measured increases of VISA-H with treatment $(\mathrm{P}<0.0001)$. The MCID was met in a majority of patients who received either radial $(62.5 \%)$ or combined treatment $(56.5 \%)$.

Conclusions. Overall findings suggest radial and combined shockwave treatment with physical therapy exercises can be effective in the management of proximal hamstring tendinopathy in runners.

\section{KEY WORDS}

Athletes; C-SWT; extracorporeal shockwave therapy; functional outcome; nonoperative treatment; proximal hamstring tendinopathy; R-SWT; sports medicine. 


\section{BACKGROUND}

Proximal hamstring tendinopathy is a common overuse injury that affects athletes and the general population. The injury may present as an insidious and progressive deep buttock pain localized to the ischial tuberosity that often worsens at initiation of activity, during acceleration running/sprinting, and with prolonged sitting. Athletes who participate in distance running, sprinting, and endurance sports are frequently affected (1). Non-athletes who develop proximal hamstring tendinopathy often have an occupational or lifestyle history of movements involving repetitive hip flexion that compressively load the proximal hamstrings $(2,3)$. Similar to other tendinopathies, proximal hamstring tendinopathy is commonly a chronic degenerative condition that arises from mechanical overload and repetitive stretch leading to cumulative tendon microtraumas $(1,4)$. In most cases, the diagnosis is made clinically by obtaining a careful history and physical examination $(1,3)$. In cases when the diagnosis is unclear or there is concern for more advanced tendon disease, magnetic resonance imaging (MRI) may be obtained; MRI can demonstrate peritendinous edema, bone marrow edema at the ischial tuberosity, and tendon thickening or degeneration along with presence of tendon tear (5). Initial management of proximal hamstring tendinopathy consists of a multimodal approach including activity modification, analgesics/anti-inflammatory medications, and physical therapy focusing on core and lumbopelvic stabilization with eventual progression to eccentric strengthening (6). Ultrasound-guided corticosteroid peritendinous injections, platelet rich plasma (PRP) injections, and extracorporeal shockwave therapy (ESWT) are alternative treatment options considered for proximal hamstring tendinopathy ( 7 , 8). Image guided peritendinous corticosteroid injections have been shown to provide short-term improvement, but longterm benefits are often not sustained, and tenotoxicity may limit use (5). PRP offers better long-term pain and functional improvements with case series demonstrating $63-68 \%$ of patients having sustained relief at 6 months post-treatment $(8,9)$. One key limitation in PRP protocols is that they typically require six weeks or greater time away from running or impact activities to allow for initial tendon healing (8).

ESWT is a non-invasive intervention that has been evaluated in running populations with good response across lower extremity injuries (10). Shockwaves are typically produced using either radial shockwave therapy (R-SWT) using a pneumatic or ballistic device or focused shockwave therapy (F-SWT) using electromagnetic, electrohydraulic, or piezoelectric sources (11). The limited research on the topic evaluated R-SWT for proximal hamstring tendinopathy by Cacchio et al., who conducted a randomized control trial in
40 professional athletes. R-SWT outperformed standard therapeutic exercise program with $80 \%$ of athletes assigned to R-SWT returning to pre-injury level of sports participation compared with no athletes in the standard treatment cohort, and benefits in the R-SWT cohort were sustained to one year (7). While most shockwave literature evaluates R-SWT or F-SWT in isolation, more recent studies have shown positive results when using both devices in a single treatment session, termed combined shockwave therapy (C-SWT) (12, 13). Studies evaluating F-SWT or C-SWT have not been reported on management of proximal hamstring tendinopathy nor have results been measured using the Victorian Institute of Sport Assessment - Proximal Hamstring Tendons (VISA-H) questionnaire.

The objective of this quality improvement study was to evaluate functional outcomes of R-SWT and C-SWT for the management of proximal hamstring tendinopathy in runners. We hypothesized that patients who received R-SWT or C-SWT would experience improvement in functional outcomes using the VISA-H.

\section{MATERIALS AND METHODS}

This report is from results of a quality improvement initiative approved by the Department of Physical Medicine and Rehabilitation with waiver of Institutional Review Board approval. SQUIRE-2 guidelines were used for reporting quality improvement data (14). Patient characteristics, treatment measures, and functional outcomes collected as standard of care were extracted using chart review from August 2017 to March 2021 by five authors (D.M.R., S.D., A.S.T., P.H.Y, C.R.) in all patients receiving R-SWT or C-SWT for the management of proximal hamstring tendinopathy. All treatments were performed at the senior authors' outpatient sports medicine clinic (A.S.T). The study was written in compliance with the international and ethical standards of Muscles, Ligaments, and Tendons Journal (15).

The diagnosis of proximal hamstring tendinopathy was determined by senior author (A.S.T.) based on history and physical examination. Prior MRIs were also reviewed, and in some cases, MRI was obtained prior to treatment as clinically determined. Inclusion criteria were the following: 1) primary diagnosis of proximal hamstring tendinopathy and 2) completed baseline and follow-up functional outcome measures VISA-H. Exclusion criteria were the following: 1) previous proximal hamstring tendinopathy surgery, 2) diagnosis of connective tissue or inflammatory disease (e.g., rheumatoid arthritis), 3) concurrent treatment of a separate lower extremity injury using shockwave therapy (e.g., plantar fasciitis, piriformis mediated pain, Achil- 
les tendinopathy, medial tibial stress syndrome), 4) referred pain from the lumbar spine, and 5) non-runners.

\section{Treatment procedure}

Patients received R-SWT or C-SWT. The clinic performing shockwave therapy offered R-SWT from August 2017 to January 2019. C-SWT was introduced as a treatment option beginning in January 2019. Since ESWT is not covered by insurance in the United States, patients paid a one-time fee for shockwave treatment sessions that was the same for both R-SWT and C-SWT. ESWT sessions occurred once a week for a minimum of four weeks based on the prior study by Cacchio $e t$ al. (7). Follow-up visits were scheduled at 6-8 weeks following the initial series of four treatment sessions. At these followup visits, additional sessions were offered on a case-by-case basis to maximize clinical outcomes. Patients who received R-SWT initially and reported unsatisfactory outcomes were offered the option to receive C-SWT at no additional cost.

R-SWT treatments were provided using the Storz Extracorporeal pulse activation technology $\left(\right.$ EPAT $\left.^{\circledR}\right)$ device (Storz Medical, Tägerwilen, Switzerland). Two applicator heads were used for each treatment, with a minimum of 3000 strikes per head at $15 \mathrm{~Hz}$ and a minimum pressure of $2.5 \mathrm{Bar}$ (range used in patient cohort: 2.5-5.0 Bar). During R-SWT, the applicator heads were positioned over the affected area using the clinical focusing technique, with careful attention to treat over the hamstring tendon origin and over other affected area/s (such as the myotendinous junction and muscles of the biceps femoris, semimembranosus and semitendinosis).

C-SWT treatments involved consecutive application of R-SWT and F-SWT in each treatment session. F-SWT sessions were conducted with the Storz Duolith device (Storz Medical, Tägerwilen, Switzerland) set to a minimum of 1000 shocks with an energy intensity minimum of $0.12 \mathrm{~mJ}$ (range used in patient cohort: 0.12-0.5 mJ). During C-SWT treatment, F-SWT targeted the proximal hamstring origin at the ischial tuberosity, and R-SWT was primarily applied over the myotendinous junction and muscles with both modalities using the clinical focusing technique.

No local or regional anesthetic was used. All patients reported pain while receiving shockwave therapy. Patients were instructed to avoid NSAIDs and icing the affected area until completion of the treatment course. Patients were allowed to resume or continue regular activities, including running, as tolerated with three days of initial rest recommended following first session of C-SWT. Physical therapy was prescribed to each patient with no prior treatment. Those who completed an extensive course of physical therapy were recommended to complete their home exercise program focusing on core/pelvic strengthening and control, progressive hamstring strengthening (concentric advanced to eccentric), gastrocnemius/soleus stretching, and deep tissue massage along the hamstring origin for soft tissue mobilization. Patients were encouraged to perform the prescribed exercise program in conjunction with ESWT treatment and throughout follow-up.

\section{Outcome assessment}

VISA-H questionnaires were collected on initial treatment day and repeat measures were obtained periodically including after completion of treatment series (typically treatment 4) and after follow-up visits. The VISA-H uses self-report on level of physical impairment from an eight-item questionnaire, covering three domains of pain, function, and sporting activity. The sporting activity section assesses the patient's ability to perform more difficult activities and is population-specific to athletes. The first six questions (1-6) concern pain and function, while the last two questions (7-8) concern sporting activities. Questions 1-7 use a 0-10 numerical rating scale, while question 8 is rated out of 30 points, to give a total summation of 100 points as the maximum attainable score. A higher score corresponds to greater physical ability; a symptomatic patient would score lower, with the minimum score being 0 points (16). Adverse outcomes were monitored during treatments and follow-up visits.

\section{Statistics}

Descriptive statistics were presented as mean and standard deviations for continuous data and frequencies with percentages for categorical data. Demographic data were compared using Welsh's t-tests (continuous), chi-squared tests (categorical for cell values greater than 5), or Fischer's exact tests (categorical for cell values less than or equal to 5); p-values were not specifically reported, as none were significant. Outcome values were evaluated between R-SWT and C-SWT mean differences and the number of patients who met criteria for clinical response using minimal clinical important difference (MCID) value of $\geq 22$ point (16). Categorical outcomes whether a patient met MCID between R-SWT and C-SWT were compared using chi-squared tests. VISA-H data were compared using mixed-design ANOVA to compare the overall effect of ESWT and to measure differences between R-SWT and C-SWT. Data was tested for normality and homogeneity of variance and covariance prior to analysis. For those treated with R-SWT who later received C-SWT, outcomes used were based on their final VISA-H score following last R-SWT treatment. All statistical analyses used two-tailed tests, and a threshold of $\mathrm{p}<.05$ was considered significant; calculations were performed in $\mathrm{R}$ ( $\mathrm{R}$ Core Team (2021), Vienna, Austria). 


\section{RESULTS}

Chart review of a single provider (A.S.T.) who performed shockwave therapy in clinic identified 90 patients who had proximal hamstring tendinopathy that were treated with either R-SWT or C-SWT during the study period (figure 1). Eighteen of these patients were excluded for additional lower extremity pathologies treated concurrently; one was excluded for systemic or rheumatologic diagnoses; one was excluded due to concurrent referred pain from the lumbar spine; seven were excluded due to non-runner status. This resulted in 63 patients eligible for inclusion.

Demographic and clinical characteristics demonstrate the population treated was on average $42.8 \pm 14.7$ years old and had symptoms for $16.9 \pm 23.8$ months (table I). Overall,
$84.1 \%$ of patients completed prior formal physical therapy before initiating ESWT.

Figure 1. Patient inclusion flowchart.

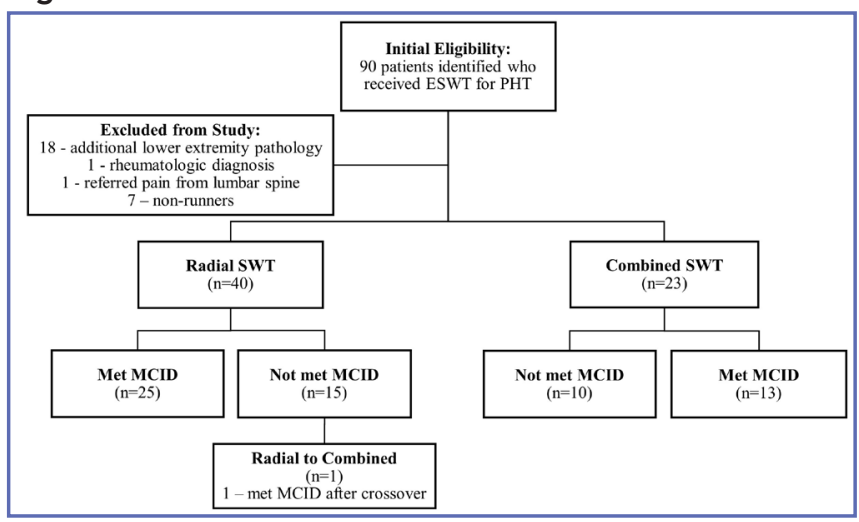

Table I. Demographic and clinical characteristics.

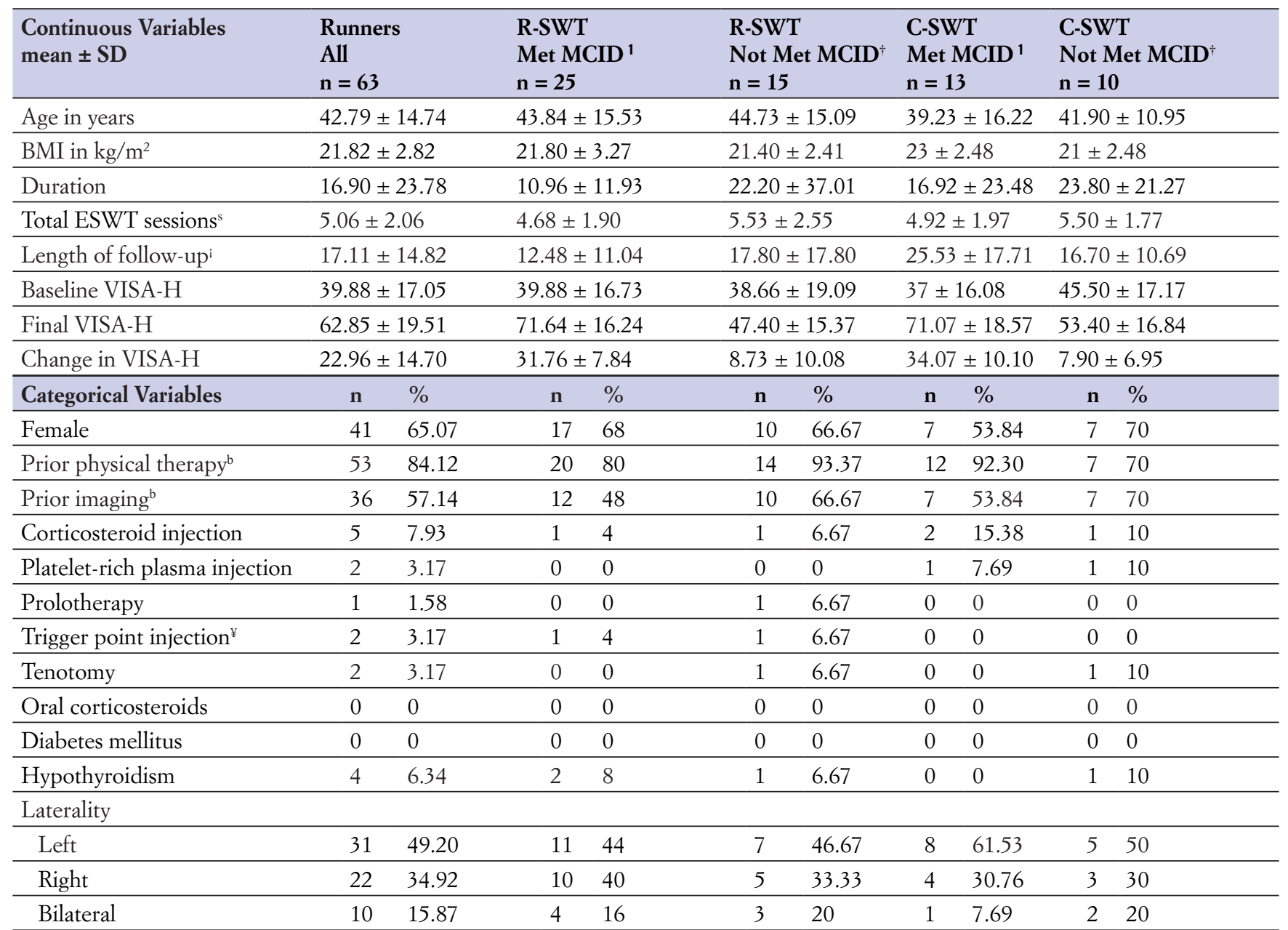

${ }^{1}$ Met MCID equals a 22-point change or greater on the VISA-H; ${ }^{+}$Not Met MCID equal a 21-point change or fewer on the VISA-H; ${ }^{\text {sTotal number of }}$ ESWT sessions from baseline VISA-H to final VISA-H; iLength of follow up in weeks from initial intake evaluation to final VISA-H; bPrior being before

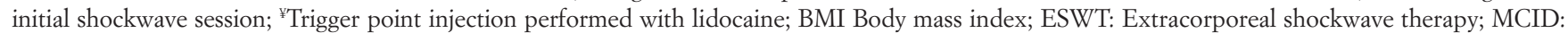
Minimal clinically important difference; SD: Standard deviation; VISA-H: Victorian Institute of Sports Assessment - Proximal Hamstring Tendons. 
Characteristics were similar between R-SWT and C-SWT cohorts (table I). VISA-H scores were similar at baseline between R-SWT and C-SWT groups (39.4 \pm 17.4 vs $40.7 \pm 17.0)$ and were not different in VISA-H scores between R-SWT and C-SWT groups following treatment (62.6 \pm 19.7 vs $63.4 \pm 21.3 ; \mathrm{p}=0.812)$. Within the entire cohort, VISA-H scores significantly increased from baseline to follow-up for both treatment groups ( $p<0.0001$, figures 2,3 ). Most patients met MCID in both R-SWT (25 of 40,62.5\%) and C-SWT (13 of 23, $56.5 \%$ ), but there was no difference between the proportion of patients that met MCID between R-SWT and C-SWT ( $\mathrm{p}=0.641)$. The mean number of sessions until patients first met MCID was $3.8 \pm 0.9$ for R-SWT and $4.5 \pm 2.0$ for C-SWT. For all the patients who met MCID, all who received R-SWT $(\mathrm{n}=25)$ and almost all treated with C-SWT (12 of $13,92.3 \%$ ) did so within six treatment sessions (figure 4).

One patient who was initially treated with R-SWT but desired additional functional gains elected to complete C-SWT and met MCID after receiving C-SWT. There was no report of major adverse reactions to shockwave therapy during treatment or follow up for both groups.

\section{DISCUSSION}

The purpose of this quality improvement report was to evaluate the functional outcomes using R-SWT and C-SWT in runners with proximal hamstring tendinopathy quantified using VISA-H. We observed that most patients met criteria for clinical improvements following R-SWT or C-SWT. No major complications were observed. To our knowledge, no studies have previously compared these two forms of shockwave therapy nor reported on outcomes after C-SWT for proximal hamstring tendinopathy management in a running population. These findings suggest runners with proximal hamstring tendinopathy may achieve functional gains with either ESWT treatment method.

Our findings are consistent with the existing literature on ESWT for proximal hamstring
Figure 2. Radial Shockwave Cohort VISA-H Score Changes.

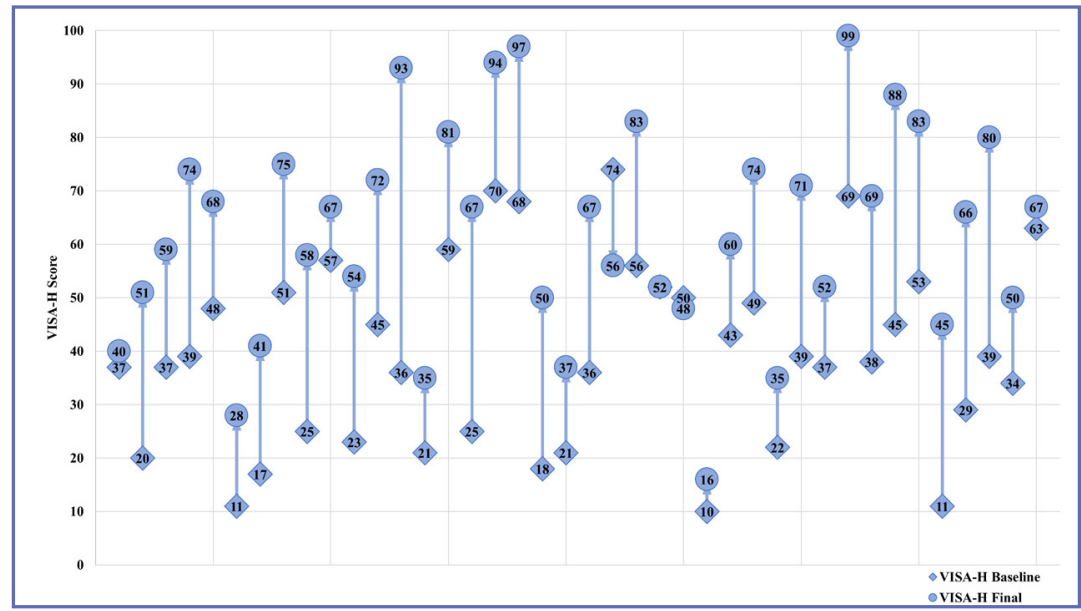

Figure 3. Combined Shockwave Cohort VISA-H Score Changes.

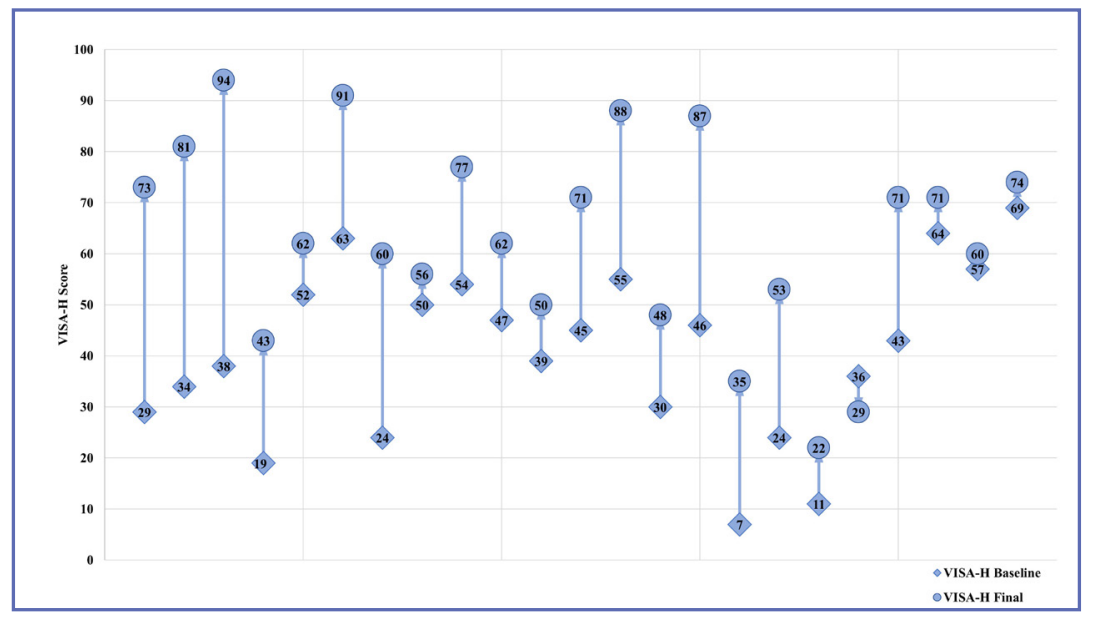

Figure 4. Number of treatments to minimal clinically important difference in radial and combined shockwave groups.

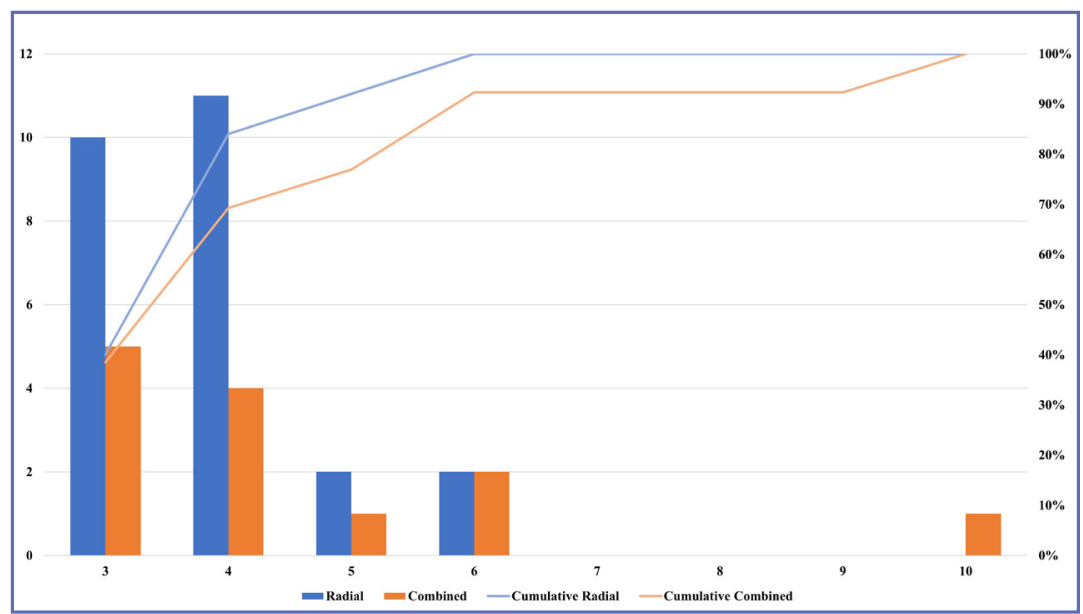

Muscles, Ligaments and Tendons Journal 2021;11 (4) 
tendinopathy. Cacchio et al. conducted a randomized control trial of 40 professional athletes with chronic proximal hamstring tendinopathy and found R-SWT to outperform standard therapeutic exercise at three months with a primary outcome of pain scores (7). The R-SWT group received four weekly treatment sessions using 2,500 strikes at 4 Bars, which equivalates to an energy flux density (EFD) of $0.18 \mathrm{~mJ} /$ $\mathrm{mm}^{2}$. Notably $80 \%$ of athletes in the R-SWT and no participants receiving therapeutic exercises were able to return to pre-injury status. We observed a majority of patients responded to R-SWT and C-SWT using a different validated outcome measure specific to athletes with the VISA-H. Our findings expand on prior overlapping cohort from a smaller case series by Mitchkash et al., which noted clinically important VISA-H score improvements in $69 \%$ of runners treated with R-SWT for proximal hamstring tendinopathy (10).

The form of ESWT did not result in differences in functional outcome measures. Limited studies to date have compared C-SWT to R-SWT. One study demonstrated that treatment of Achilles tendinopathy had more favorable outcomes measured with higher number of patients meeting MCID who received C-SWT compared to R-SWT (89.7\% vs $63.8 \%, p=0.022)(17)$. In contrast, a separate report in the treatment of plantar fasciitis with C-SWT to R-SWT observed similar gains with nearly three-quarters of patients meeting MCID using the Foot and Ankle Ability Measure (FAAM) in both forms of treatment (13). Notably higher total EFD delivered during treatment may be more important than treatment energy intensity levels for obtaining successful outcomes (18). While we did not directly measure total EFD, the mean number of strikes (6000) with a goal to aim for 4 bars (similar to the energy level achieved by Cacchio) may contribute to a high total EFD in those treated with R-SWT. While it has not been specifically evaluated in proximal hamstring tendinopathy, it is plausible that total EFD received explains our similar high success rates between groups. In addition, we aimed to highlight the safety profile of ESWT for proximal hamstring tendinopathy. No major complications were observed in our cohort, similar to prior reports (7).

While this study is the first to compare R-SWT and C-SWT for proximal hamstring tendinopathy in a running cohort,

\section{REFERENCES}

1. Chu SK, Rho ME. Hamstring injuries in the athlete: Diagnosis, treatment, and return to play. Curr Sports Med Rep 2016;15(3):184-90.

2. Lempainen L, Sarimo J, Mattila K, Vaittinen S, Orava S. Proximal hamstring tendinopathy: Results of surgical we recognize limitations in our report. All enrolled patients were runners, which may limit generalizing findings to other athlete populations or those who are less active. The quality improvement study design limits use of randomization, and we did not have a control group to account for influence of physical therapy on functional gains. However, most patients had prior physical therapy and reported a mean duration of symptoms exceeding a year which suggests gains in VISA-H are unlikely to be primarily due to spontaneous healing. We could not control for the specific physical therapy exercises or frequency/compliance to performing this program. Furthermore, ESWT is not covered by most insurers in the United States for musculoskeletal injuries, and patients incurred out of pocket costs which may introduce potential for bias and limit generalizing findings.

\section{CONCLUSIONS}

In summary, our findings suggest most runners with proximal hamstring tendinopathy benefit from both R-SWT and C-SWT, and no major complications were observed for either group. Practical application of findings suggests both R-SWT and C-SWT may be helpful in the management of proximal hamstring tendinopathy when combined with physical therapy exercises, and six sessions of treatment may be required to optimize response. These results may aide in the development of future randomized controlled trials or prospective cohort studies evaluating the use of shockwave therapy use in management of proximal hamstring tendinopathy.

\section{ETHICS}

Approval was obtained from our institution's quality improvement advisory board; IRB approval was thereby waived by the institution. The letter of approval can be provided if requested.

\section{CONFLICT OF INTERESTS}

The authors declare that they have no conflict of interests.

management and histopathologic findings. Am J Sports Med 2009;37(4):727-34.

3. Lempainen L, Johansson K, Banke IJ, et al. Expert opinion: Diagnosis and treatment of proximal hamstring tendinopathy. Muscles Ligaments Tendons J 2015;5(1):23-8. 
4. Goom TSH, Malliaras P, Reiman MP, Purdam CR. Proximal hamstring tendinopathy: Clinical aspects of assessment and management. J Orthop Sports Phys Ther 2016;46(6):483-93.

5. Zissen MH, Wallace G, Stevens KJ, Fredericson M, Beaulieu CF. High hamstring tendinopathy: MRI and ultrasound imaging and therapeutic efficacy of percutaneous corticosteroid injection. Am J Roentgenol 2010;195(4):993-8.

6. Fouasson-Chailloux A, Menu P, Meslan O, Guillodo Y, Crenn V, Dauty M. Evolution of isokinetic strength and return to sport after proximal hamstring rupture without surgical repair: a retrospective series of cases. Muscles Ligaments Tendons J 2019;9(2):173-80.

7. Cacchio A, Rompe JD, Furia JP, Susi P, Santilli V, De Paulis F. Shockwave therapy for the treatment of chronic proximal hamstring tendinopathy in professional athletes. Am J Sports Med 2011;39(1):146-53.

8. Auriemma MJ, Tenforde AS, Harris A, McInnis KC. Platelet-rich plasma for treatment of chronic proximal hamstring tendinopathy. Regen Med 2020;15(4):1509-18.

9. Fader RR, Mitchell JJ, Traub S, et al. Platelet-rich plasma treatment improves outcomes for chronic proximal hamstring injuries in an athletic population. Muscles Ligaments Tendons J 2014;4(4):461-6.

10. Mitchkash M, Robinson D, Tenforde A. Efficacy of Extracorporeal Pulse-Activated Therapy in the Management of Lower-Extremity Running-Related Injuries: Findings From a Large Case Cohort. J Foot Ankle Surg 2020;59(4):795-800.

11. Reilly JM, Bluman E, Tenforde AS. Effect of Shockwave Treatment for Management of Upper and Lower Extremity Musculoskeletal Conditions: A Narrative Review. PM R 2018;10(12):1385-403.
12. Vahdatpour B, Mokhtarian A, Raeissadat S, Dehghan F, Nasr N, Mazaheri M. Enhancement of the Effectiveness of Extracorporeal Shock Wave Therapy with Topical Corticosteroid in Treatment of Chronic Plantar Fasciitis: A Randomized Control Clinical Trial. Adv Biomed Res 2018;7:62.

13. DeLuca S, Robinson DM, Yun PH, Rosenberg C, Tan CO, Tenforde AS. Similar Functional Gains Using Radial Versus Combined Shockwave Therapy in Management of Plantar Fasciitis. J Foot Ankle Surg 2021;S1067-2516(21)00130-7.

14. Ogrinc G, Davies L, Goodman D, Batalden P, Davidoff F, Stevens D. SQUIRE 2.0 (Standards for Quality Improvement Reporting Excellence): Revised publication guidelines from a detailed consensus process. BMJ Qual Saf 2016;25(12):986-92.

15. Padulo J, Oliva F, Frizziero A, Maffulli N. Muscles, Ligaments and Tendons Journal - Basic principles and recommendations in clinical and field Science Research: 2018 update. Muscles Ligaments Tendons J 2018;8(3):305-7.

16. Cacchio A, De Paulis F, Maffulli N. Development and validation of a new visa questionnaire (VISA-H) for patients with proximal hamstring tendinopathy. Br J Sports Med 2014;48(6):448-52.

17. Robinson DM, Tan CO, Tenforde AS. Functional Gains Using Radial and Combined Shockwave Therapy in the Management of Achilles Tendinopathy. J Foot Ankle Surg 2021:S1067-2516(21)00226-X.

18. Chang KV, Chen SY, Chen WS, Tu YK, Chien KL. Comparative effectiveness of focused shock wave therapy of different intensity levels and radial shock wave therapy for treating plantar fasciitis: A systematic review and network meta-analysis. Arch Phys Med Rehabil 2012;93(7):1259-68. 